\title{
Article \\ Determination of the modulus of elasticity using a Smartphone and mode of unsupport beam Euler-Bernoulli
}

\author{
Newton Huamaní ${ }^{1, \dagger, \ddagger(D 0000-0002-9447-2556 *}$ \\ $1 \quad$ E-mail; newton.h.c@hotmail.com \\ * Current address: Perú \\ $\dagger$ Profession: Graduate in Physics-Mathematics \\ + Others: Master in Education.
}

\begin{abstract}
This paper presents the simplified and quick way to obtain an experimental measure of the modulus of elasticity(E) of a piece of metal rod using the Smartphone microphone and through the experimental configuration in mode of unsupport beam Euler-Bernoulli(E-B). To understand it, it is required to have knowledge about flexural vibration of E-B beams and to know the analysis of the spectogram of the audio signal. The methodology consists in measuring the flexural resonance frequency through an app installed on a Smartphone and using the $E$ formula deduced from the E-B theoretical framework. The results of the experimental measurements have a discrepancy of less than $5 \%$ with respect to the factory value, for a carbon steel rod with circular cross section using the impact excitation technique. It describes the precision and accuracy of the experimental measurement. It is useful, because it helps to classify the materials in the Faculty of Engineering and it helps to make a more efficient quality control.
\end{abstract}

Keywords: Modulus of elasticity 1; frequency spectrum 2; mic 3; Euler-Bernoulli equation 4

\section{Introduction}

The Smartphone recently are instruments of daily use of a student and teacher in the learning and teaching of Mathematics and Physics, and these mobile devices allow a wide range of measurements through their internal sensors just by installing a free app [1].

This paper presents the simplified and quick way to obtain an experimental measure of the modulus of elasticity(E) of a piece of metal rod using the Smartphone microphone and through the experimental configuration in mode of unsupport beam Euler-Bernoulli(E-B). To understand it, it is required to have knowledge about flexural vibration of E-B beams and to know the analysis of the spectogram of the audio signal.

The methodology consists in measuring the flexural resonance frequency through an app installed on a Smartphone and using the $E$ formula deduced from the $E-B$ theoretical framework. The results of the experimental measurements have a discrepancy of less than $5 \%$ with respect to the factory value, for a carbon steel rod with circular cross section using the impact excitation technique.

The $E$ is a physical parameter that shows the capacity of the material to resist linear elastic deformation and is obtained from the quotient between tensile stress(direct stress over area) and tensile strain, in its linear elastic behaviour.

The main tests to measure $E$ are: linear stretching, which consists of measuring longitudinal elongation after release of the applied direct tensile stress; another is based on the use of the mechanical vibrator to find the resonances [2]; another is based on first measuring the speed of propagation of the waves, [3], and then this makes it possible to calculate the elasticity of the material; another is based on the use of data acquisition systems (use of sensors) to find the fundamental frequency [4].

It is useful, because it helps to classify the materials in the Faculty of Engineering and it helps to make a more efficient quality control.The work is divided into sections: introduction, theoretical foundation, measurement plan, and measurement results.

\section{Theoretical fundament and formula}

The vibration of a rod arranged in mode of unsupport beam E-B, see Figure 1(a), belongs to the vibration of a continuous elastic body, and is capable of being both transverse and longitudinal, 
and also when really vibrates is a dispersive medium. Consequently, its vibration can be expressed as the superposition of several (or infinite) transversal modes.

The theoretical model, for our case, assumes that both ends, $z=0$ and $z=L$, of the bar are free (figure 1(a)), it is also uniform along its length , of constant cross-sectional area, elastic linear material, isotropic, homogeneous, its transverse dimensions are much smaller than its length, considering only small flexural deformations, its unsupport damping vibration is described by the Euler-Bernoulli equation [2]

$$
\frac{E I}{\rho A} \frac{\partial^{4} u(z, t)}{\partial z^{4}}+\frac{\partial^{2} u(z, t)}{\partial t^{2}}=0
$$

where: $u(z, t)[m]$ is the displacement of the rod at the point $z[m]$ in the instant $t[s] ; E\left[N / m^{2}\right]$ is modulus of elasticity; $I\left[\mathrm{~m}^{4}\right]$, the second moment of the transverse-section of its longitudinal axis; $\rho\left[K g / m^{3}\right]$, rod mass density; $A\left[\mathrm{~m}^{2}\right]$, the rod transvere-section.

The boundary conditions for the end $z=0$ are

$$
\frac{\partial^{2} u(0, t)}{\partial z^{2}}=0 \quad \text { and } \quad \frac{\partial^{3} u(0, t)}{\partial z^{3}}=0,
$$

while at the end $z=L$ are

$$
\frac{\partial^{2} u(L, t)}{\partial z^{2}}=0 \quad \text { and } \quad \frac{\partial^{3} u(L, t)}{\partial z^{3}}=0 .
$$

Solving the equation (1) by the variable separation method and making use of the boundary conditions (2) y (3) is obtaine $\cos (q L) \cdot \cosh (q L)=1$ and simplifying to the convenient way

$$
\tan \left(\frac{q L}{2}\right)= \pm \tanh \left(\frac{q L}{2}\right)
$$

where $q^{4}=\left(\rho A \omega^{2}\right) / E I$.

Finding the roots of the equation (4) we obtain the resonant frequencies of modes(peak) of flexural vibration, given by

$$
f_{n}^{2}=\frac{E I q_{n}^{4}}{4 \pi^{2} \rho A} \quad(n=1,2,3, \cdots),
$$

where in the equation (5): $q_{1} L=(\pi / 2) 3.0112, q_{n} L=(\pi / 2)(2 n+1)$ con $n=2,3,4, \cdots$.

Inasmuch as $I=A(d / 4)^{2}$ and solving $E$ from equation (5). Therefore, the formula of the modulus of elasticity, for all the peaks or resonant frequencies, of the rod of circular cross section of diameter $d[m]$ of mass $m[K g]$ and length $L[m]$ is

$$
E=\frac{2^{12}}{\pi^{3}} \frac{m L^{3}}{d^{4}} f_{n}^{2}\left(\frac{1}{3.0112^{4}} ; \frac{1}{5^{4}} ; \frac{1}{7^{4}} ; \frac{1}{9^{4}} ; \cdots\right),
$$

where $f_{n}[H z]$ is the $\mathrm{n}$-th resonant frequency.

Remark 1.- if we do $v=\sqrt{E / \rho}$ in equation (5) we obtain the relationship between frequency and length ${ }^{1}$ for unsupported cylindrical rods

$$
f=\frac{v \pi d}{32 L^{2}}\left(3.0112^{2} ; 5^{2} ; 7^{2} ; 9^{2} ; \cdots\right)
$$

Remark 2.- You can also get $E$, holding the rod at its midpoint and impacting axially at one end to establish a longitudinal vibration and the formula to use in this case would be $E=4 \rho L^{2} f_{0}^{2}$ where $L$ is length, $\rho$ is density, $f_{0}$ is the fundamental or longest longitudinal resonance frequency [4]. The drawback is that longitudinal waves in rods with corrugations, their corrugations constitute reflective points for the wave.

1 Result that is analogous to the reference[2] in your strapping vibrations section, page 3. 


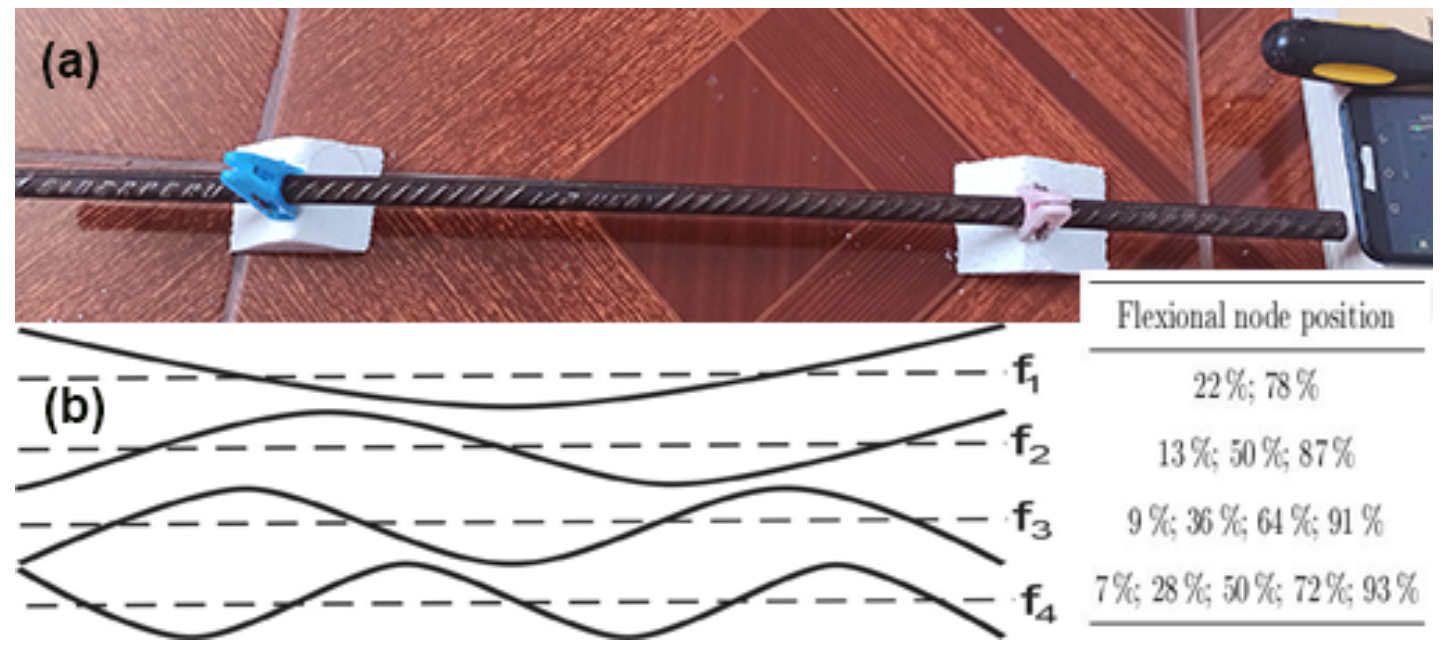

Figure 1. (a) Experimental setup: mode of unsupport beam Euler-Bernoulli; (b) Diagram of the first four way of flexural vibration $\left(f_{1}, f_{2}, f_{3}, f_{4}\right)$

\section{Measurement plan}

The determination of $E$ is subject to the arrangement of the rod in unsupported beam mode E-B and is subject to the equation (6). The magnitude that requires attention is the resonant frequencies, therefore, the main job is to obtain the frequency spectrum of the impact audio signal in such a way that the peaks can be easily identified. The assembly of the equipment and its configuration must be such as to minimize random and systematic errors [5]; in addition, the rod must have a flexural vibration that reflects its structure and inherent dynamic characteristics.

The experimental setup is shown in Figure 1(a) and the diagram of the first four modes of flexural vibration is shown in Figure 1(b)

Measurement materials and instruments: Smartphone with microphone; a corrugated carbon steel rod for reinforced concrete reinforcement(ASTM A615-1/2N60 with deformation 0.0021) [6]; polystyrene insulators and a plastic hammer to impact, the mass of which does not exceed $5 \%$ of the rod; formula deduced from the theoretical framework E-B (equation (6)); vernier; digital balance; rule.

The theoretical value of $\mathrm{E}$ is $200 \pm 7 \mathrm{GPa}$, it was obtained from the Manufacturing Technical Sheet [6].

Procedure: (1st) measure the length, diameter, mass of the rod; (2nd) capture the outstanding peaks or resonant frequencies with the help of the Smartphone provided with the FFT Spectrum Analyzer app downloaded for free from the Play Store, impacting in the center with the hammer; (3rd) replace the values of the outstanding peaks or resonant frequencies in their respective formulas (note that the equation 6 goes from lowest to highest resonant and the constant for the fundamental is decimal and for the rest they are integers). Suggestions: rest the rod in its nodes on extruded polystyrene, to avoid coupling with external vibrations, see figure 1(a); take at least three captures of the frequency spectrum and then average or interpolate; impacting around the center at equidistant points; to interpolar consider the center as $x=0$ making the points before the center negative and positive after the center; place the sensor in the antinode at a distance less than or equal to $1 \mathrm{~cm}$; try in the absence of loud noises but without special restrictions.

Given that there are four rods of the same material in analysis of different lengths $(0.7000$; $0.6020 ; 0.5020 ; 0.4000 \pm 0.0005$ in $m$ ) the elasticity modulus will be determined using a laptop with a processor i5 and with built-in microphone provided with free Audacity2.3.3, [7]. The procedure will be to rest the rods in unsupported mode and we will bring the Laptop about $8 \mathrm{~cm}$ to record the audio and then analyze the frequency spectrum with a Nyquist-Shannon sample configuration $44100 \mathrm{~Hz}$ and depth of 16 bits to the microphone power of $+20 \mathrm{~dB}$. 


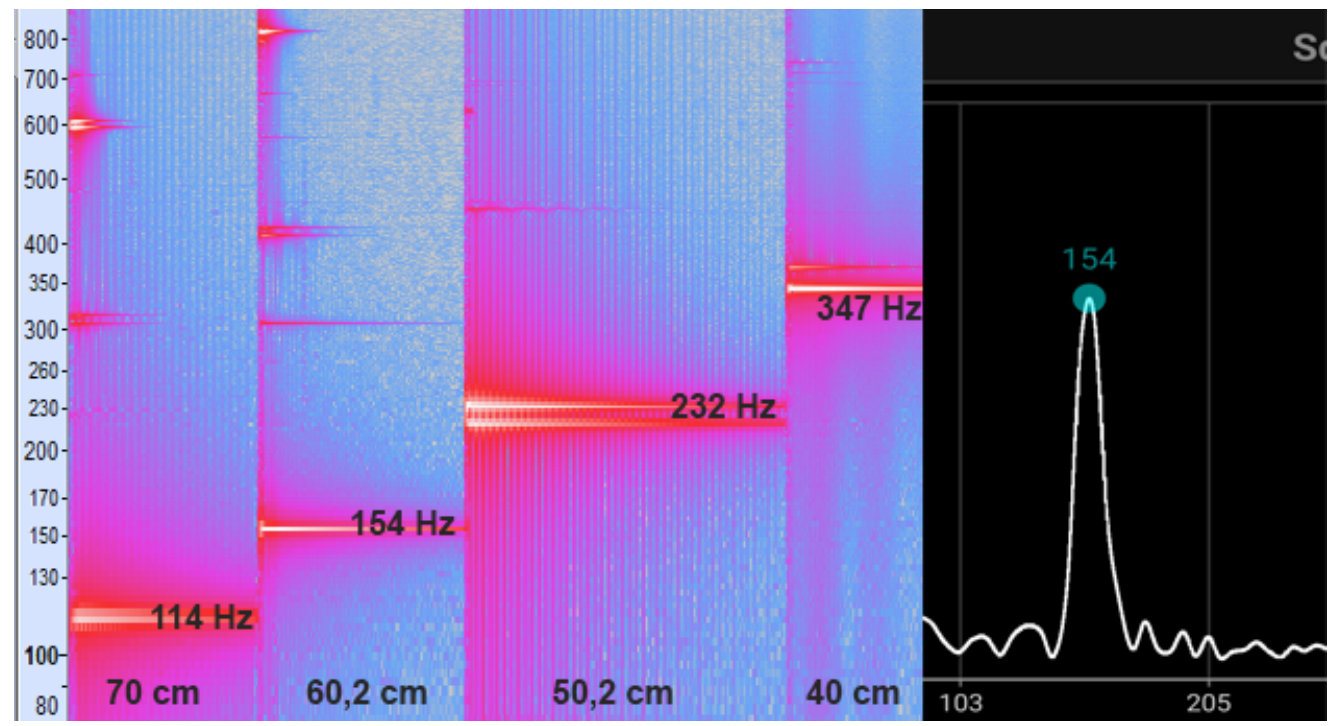

Figure 2. (Left): Impact audio signal frequency spectrogram of the four rods recorded with the integrated microphone and analyzed with Audacity2.3.3; (right): The outstanding peak is the fundamental frequency of the rod under study obtained with the FFT Spectrum Analyzer app, see annex.

\section{Measurement results and their discrepancy}

When the rod is long, its fundamental resonant frequency is too low $(<20 \mathrm{~Hz})$, making it difficult to measure; while when it is too short, the fundamental resonant frequency is high, escaping from the measurement range $(>20 \mathrm{KHz})$. So for our instruments, a length $(0.6020 \pm 0.0005) \mathrm{m}$ is reasonable.

Measurements of the rod under study: length $L=(0.6020 \pm 0.0005) \mathrm{m}$; mass $m=0.5984 \pm$ $0.0001 \mathrm{Kg}$; diameter $d=0.0127 \pm 0.0001 \mathrm{~m}$.

We capture six times the resonant peaks or frequencies with FFT Spectrum Analyzer, see annex, which we record in Table 1(right), the first $\left(f_{1}\right)$ and the second $\left(f_{2}\right)$ resonant. It was interpolated using SPSSv23(statistical software [8]) with the following results: $f_{1}^{x}=154.214+0.091 x-0.010 x^{2}-$ $0.028 x^{3}+r$ with $R^{2}=0,868$ and $f_{1}^{0}=154.214 \mathrm{~Hz} ; f_{2}^{x}=423.857-4.611 E-17 x+0.245 x^{2}+r$ with $R^{2}=0,98$ and $f_{2}^{0}=423.857 \mathrm{~Hz}$. This indicates and corroborates that the resonant frequencies $f_{1}$ and $f_{2}$ are not harmonic, in general the $\mathrm{n}$-th resonants are not harmonic.

Table 1: (left): fundamental frequencies of the four rods obtained with Audacity2.3.3; (right): resonant peaks or frequencies obtained with the FFT Spectrum Analyzer app installed on the Smartphone

\begin{tabular}{|c|c|c|c|c|c|c|}
\hline (left) & $L_{i}[m]$ & $f_{1 i}[H z]^{a}$ & (right) & $x_{i}$ & $f_{1}[H z]^{b}$ & $f_{2}[\mathrm{~Hz}]^{c}$ \\
\hline & 0.7000 & 114 & & -3 & 153 & 426 \\
\hline & 0.6020 & 154 & & -2 & 154 & 425 \\
\hline & 0.5020 & 232 & & -1 & 154 & 424 \\
\hline & \multirow[t]{4}{*}{0.4000} & 347 & & 0 & $\ldots$ & $\ldots$ \\
\hline & & & & 1 & 154 & 424 \\
\hline & & & & 2 & 155 & 425 \\
\hline & & & & 3 & 155 & 426 \\
\hline
\end{tabular}

${ }^{a}$ fundamental frequency for each length

$b$ first or fundamental frequency of the rod under study

${ }^{c}$ second resonant frequency of the rod under study

The Smartphone was configured for a sampling of Nyquist-Shannon 44100 samples and with a depth of 14 bits and accepting the uncertainty $\delta f= \pm 3 \mathrm{~Hz}$ then the interpolated fundamental resonant is cited as $f_{1}=154 \pm 3 \mathrm{~Hz}$ and replacing in the equation (6) we obtain the experimental value of the modulus of elasticity $E=191 \pm 10 \mathrm{GPa}$ for the rod under study with a discrepancy of 


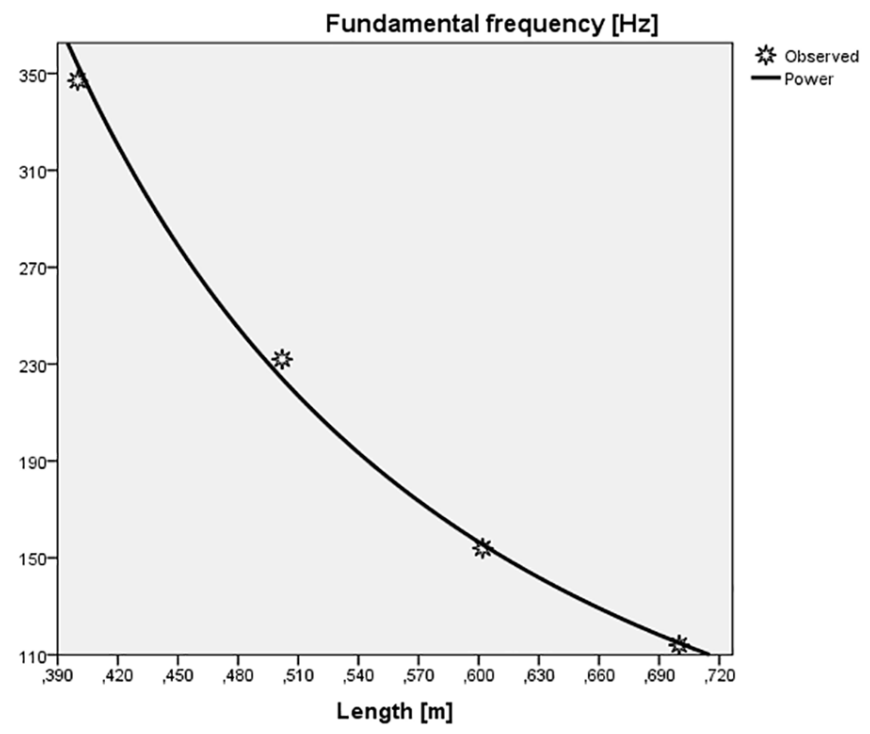

Figure 3. Behavior of $\mathrm{f}$ vs $\mathrm{L}$, linear scale: $\mathrm{f}$ is inversely proportional to a power 2 of length $\mathrm{L}$

$4.5 \%$ with respect to the theoretical value. The total uncertainty was calculated with the differentiation technique with respect to each variable and as the square root of the sum of the squares of contributions of each variable.

Results for the four rods: figure 2(left) shows the spectrogram of the audio recorded by the laptop microphone obtained with Audacity2.3.3 [7] for each rod. in table 1(left) the fundamental frequencies and lengths of the rods are recorded. The point cloud, figure 3 , suggests that the fundamental frequency is inversely proportional to a power $n$ of the length $L$, i.e., of the form $f=K L^{-n}$. Performing a regression analysis with curvilinear estimation in SPSS.v23, see figure 3, it is obtained that $f=56.094 L^{-2.009} ; R^{2}=0.998$ then we deduce $n \approx 2$.

This result shows that the resonance frequencies can be determined with the equation (7). Identifying $(v \pi d / 32) 3.0112^{2}=K=56.094$ then it follows that the speed of the longitudinal waves in the rods is $v=(4962 \pm 39) \mathrm{m} / \mathrm{s}$ where the uncertainty was obtained considering only with respect to the diameter while the software constant was taken as constant. Replacing in the formula $E=\rho v^{2}=\left(4 m / \pi L d^{2}\right) v^{2}$ we have the experimental measure of the modulus of elasticity $E=193 \pm 4 \mathrm{GPa}$ with a discrepancy of $3.5 \%$ with respect to the theoretical. This technique [3] is precise so an explanation for the decrease in modulus with respect to the technical sheet could be that the material was subjected to different temperatures. Since it is known that metals subjected to high temperatures, their elasticity decreases [4].

Holding with clothespins on the nodes, the spikes can be deleted. Accuracy can be improved by rechecking the calibration of the measuring instruments. Accuracy can be improved by reducing random uncertainties; You can go for a digital micrometer instead of a vernier to measure the diameter, you can also go for a professional condenser microphone instead of the built-in mic, you may also prefer to use the sound card drivers to eliminate noise.

\section{Discussion}

This document presents several ways to obtain an experimental measure of modulus of elasticity(E) in a simplified and fast way with easily accessible instrumentation. With the Smartphone, the experimental measure of the modulus of elasticity is $E=191 \pm 10 \mathrm{GPa}$ which has a discrepancy of less than $5 \%$ with respect to the theoretical value. The flexural resonance frequencies of the studied material are not harmonic.It is a complementary work on issues that were not said in the article by $\mathbf{J}$. Nunn(2015) [4] It is a job for university teaching as it teaches us that the proper use of concepts in mathematics, physics and metrology leads to precise and accurate results. 


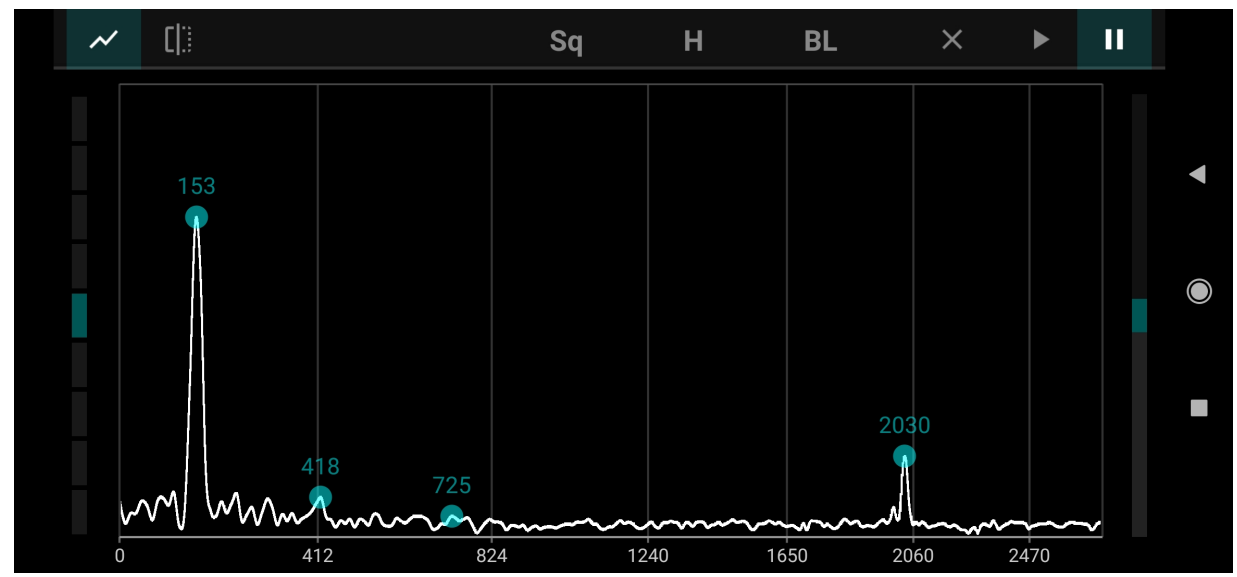

Figure A1. Spectrum analyzer interface when captured the $60.2 \mathrm{~cm}$ rod frequency

Data Availability Statement: Creator: Newton Huamani Castro ; Publication year: 2021; Title: Determination of the modulus of elasticity using a Smartphone and mode of unsupport beam Euler-Bernoulli; Audio link: https://www.dropbox.com/s/te62q5j2neavot0/Audio4RodNHC.wav?dl=0
Abbreviations
E modulus of elasticity
E-B Euler-Bernuolli
FFT Fast Fourier Transform

The following abbreviations are used in this manuscript:

\section{Appendix A. FFT Spectrum Analyser}

It is a spectrum analyzer that can be downloaded for free from the App Store, it has the ability to detect resonant frequency peaks, sliding the side buttons gives a better visualization of the peaks within the range(see figure A1), it has an adjustable scale (linear, logarithmic, etc.) and has various window functions.

\section{References}

1. Monteiro, M.; Stari, C.; Cabeza, C.; Marti, A.C. Physics experiments using simultaneously more than one smartphone sensors. Journal of Physics: Conference Series. IOP Publishing, 2019, Vol. 1287, p. 012058.

2. Repetto, C.; Roatta, A.; Welti, R. Measurements of resonant frequencies, loss factor and dynamic Young modulus of cantilever beams. Revista Brasileira de Ensino de Física 2014, 36, 1-8.

3. Hirth, M.; Gröber, S.; Kuhn, J.; Müller, A. Experimentelle Untersuchung akustischer Resonanzen in eindimensionalen Wellenträgern mit Smartphone und Tablet-PC. PhyDid A-Physik und Didaktik in Schule und Hochschule 2015, 1, 12-25.

4. Nunn, J. Measuring Young's modulus the easy way, and tracing the effects of measurement uncertainties. Physics Education $2015,50,538$.

5. Monteiro, M.; Stari, C.; Cabeza, C.; Marti, A.C. An Approach to Teach Error Analysis and Uncertainties based on Mobile-device Sensors. arXiv preprint arXiv:2005.13617 2020.

6. SiderPerú. Factory Technical Data Sheet. https://www.siderperu.com.pe/productos/barra-de-construccion visited in 2021.

7. Team, A. Audacity [free software]. http://www.audacityteam.org (Windows, MacOS, Linux) copyright 1999-2019 and last accessed June 2020, Version 2.3.3.

8. https://www.ibm.com/products/spss statistics. SPSSv23 Statistics is a powerful statistical software platform. It delivers a robust set of features that lets your organization extract actionable insights from its data $\mathbf{2 0 2 0}$. 